\title{
Pulmonary cement emboli after kyphoplasty
}

\author{
Jeffrey Forris Beecham Chick • Nikunj Rashmikant Chauhan • \\ Katherine Marie Mullen · Ryan James Bair · Bharti Khurana
}

Received: 9 July 2012/Accepted: 8 August 2012/Published online: 23 August 2012

(C) SIMI 2012

\section{Case}

A 37-year-old woman with estrogen/progesterone receptor positive breast cancer with metastases to the lungs, liver, and lumbar spine, being treated with vinorelbine, initially presented to this hospital with severe lower back pain. At the time of presentation, multiplanar magnetic resonance imaging of the spine after the administration of intravenous gadolinium demonstrated anterior wedge compression deformities of the L1 and L2 vertebral bodies (Fig. 1). The patient underwent bilateral transpedicular kyphoplasty, without evidence of cement extravasation (Fig. 2). One month later, after an uneventful recovery, the patient returned to this hospital with pleuritic chest pain, shortness of breath, and generalized weakness for 4 days. On physical examination, the patient was tachycardic with a heart rate of 115 beats per min, with diffuse chest wall tenderness and diminished breath sounds at the bilateral lung bases. Laboratory testing demonstrated an elevated

J. F. B. Chick $(\bowtie) \cdot$ N. R. Chauhan - K. M. Mullen ·

B. Khurana

Department of Radiology, Brigham and Women's Hospital, Harvard Medical School, Boston, MA 02115, USA

e-mail: jchick@partners.org

\section{R. J. Bair}

Department of Radiation Oncology,

University of Chicago Medical Center,

The University of Chicago, Chicago, IL 60637, USA
D-dimer of $1,700 \mathrm{ng} / \mathrm{mL}$ (normal reference range less than $250 \mathrm{ng} / \mathrm{mL}$ ). A portable chest radiograph demonstrated a possible serpiginous linear density within the left lung, without evidence of pneumonia, pleural effusions, or pneumothorax (Fig. 3). Given the patient's pleuritic chest pain, shortness of breath, and tachycardia, history of metastatic disease, and elevated D-dimer, computed tomography of the chest with pulmonary angiography protocol was completed to evaluate for pulmonary emboli. Analysis of the study demonstrated numerous linear hyperdensities throughout the arterial tree, consistent with pulmonary cement emboli from the prior kyphoplasty as well as multiple hypodense filling defects representing associated thrombi (Fig. 4). The patient was admitted to the Medical Oncology Service, where she was started on enoxaparin, and eventually discharged with plans to continue vinorelbine treatments. On return clinic visits 1 month later, the patient reported complete resolution of chest pain, shortness of breath, and weakness, with mild improvement in back pain.

\section{Discussion}

Vertebral body compression fractures are common, occurring at a rate of 500 per 100,000 in patients aged 50-54 and 2,960 per 100,000 in patients older than 85, and prompting numerous emergency department visits per year [1]. Initial treatment consists of conservative measures including analgesia, bed rest, and bracing. Further management of intractable pain from vertebral compression fractures may be undertaken with vertebroplasty and kyphoplasty, although these procedures remain somewhat controversial. Vertebroplasty and kyphoplasty are associated with a variety of complications including infection, 


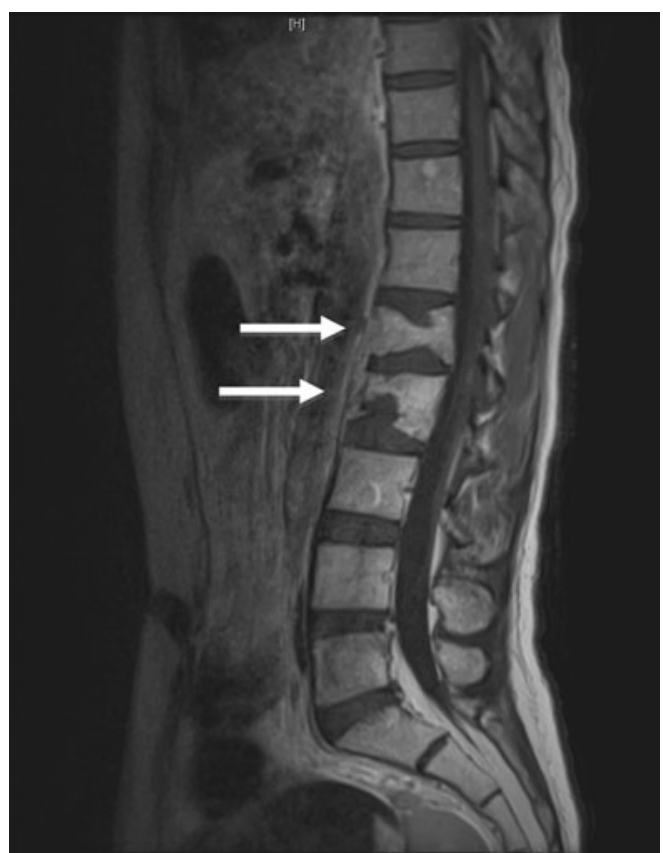

Fig. 1 Single T2-weighted sagittal image from magnetic resonance imaging of the spine demonstrating anterior wedge compression deformities of the L1 and L2 vertebral bodies (arrows)

bleeding, and increased pain and weakness post-procedurally as well as cement extravasation resulting in venous thrombosis, spinal stenosis, nerve root compression, spinal cord compression, and pulmonary cement emboli [2]. Cement extrusion has been estimated to occur in 3-70\% of vertebroplasties and 8-33\% kyphoplasties, with radiographically evident pulmonary cement emboli produced in $4.6 \%$ of cases [3, 4]. While there are no standards of care for the treatment and management of pulmonary cement emboli, asymptomatic individuals may be effectively managed conservatively with close clinical monitoring [5].

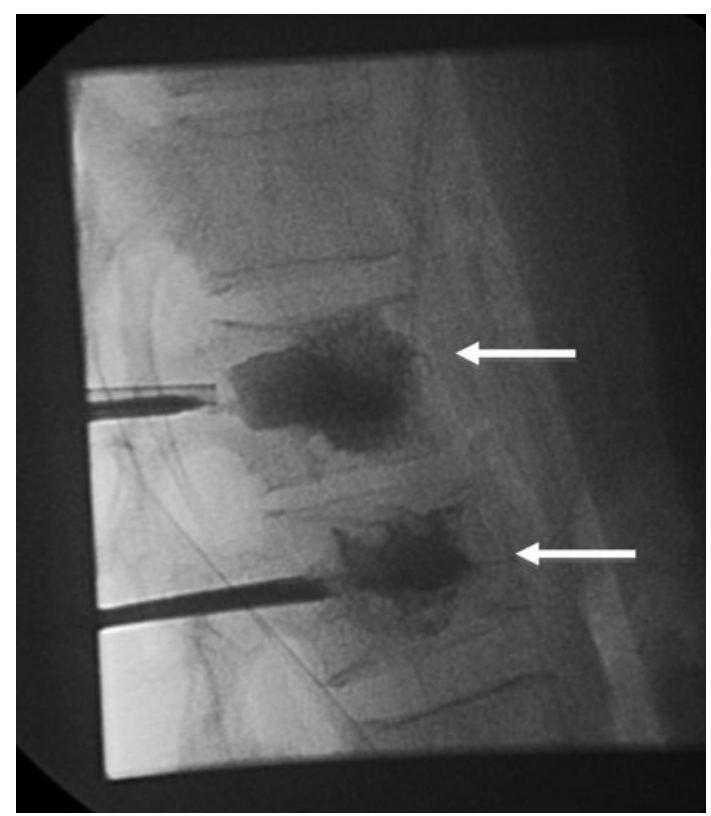

Fig. 2 Single fluoroscopic image from a bilateral transpedicular kyphoplasty demonstrating appropriate distribution of bone cement in the L1 and L2 vertebral bodies, without evidence of cement extravasation (arrows)

Symptomatic patients, on the other hand, may be managed with heparin followed by warfarin for at least 6 months, which is hypothesized to allow endothelialization of the thrombogenic cement material and prevent progressive pulmonary artery occlusion [5]. Overall, this case highlights an unusual form of pulmonary emboli and underscores the need to consider a broad differential diagnosis for chest pain and shortness of breath in the setting of recent operative interventions, including vertebroplasty and kyphoplasty. 
Fig. 3 Portable chest radiograph (left) and coneddown-view of the left hemithorax (right) demonstrating a possible serpiginous linear density within the left lung (arrows)
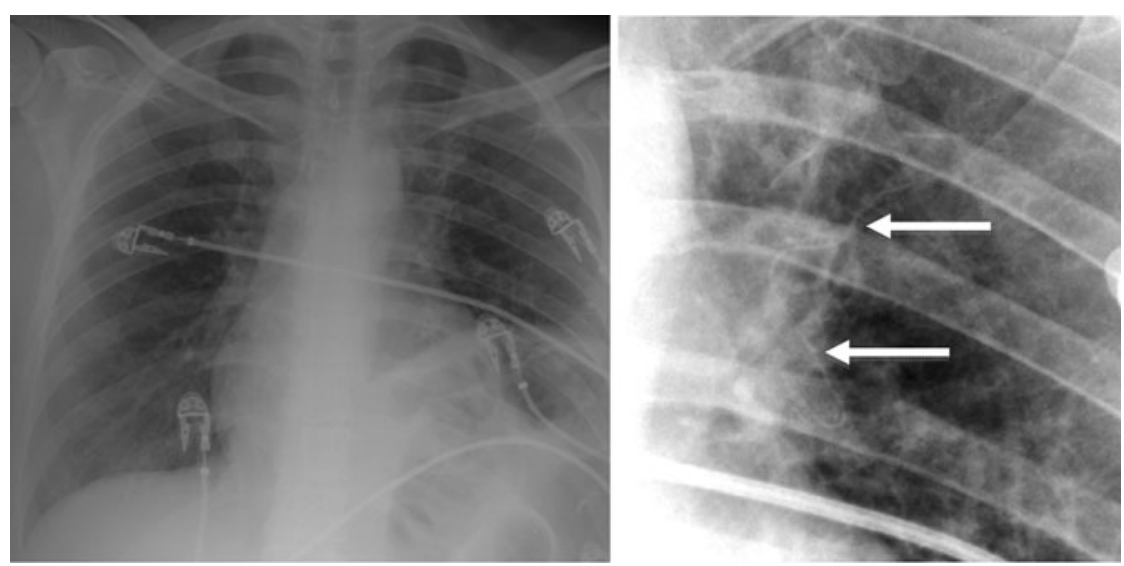

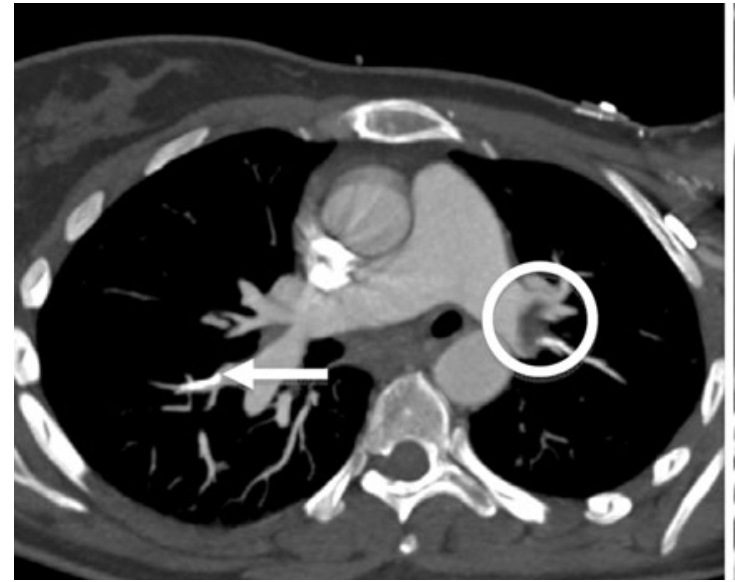

Fig. 4 Axial (left) and coronal (right) images from a computed tomography of the chest with pulmonary angiography protocol demonstrating multiple hyperdense, linear densities within the right lower lobar arteries (arrows) as well as hyperdense, linear densities

\section{Conflict of interest None.}

\section{References}

1. Melton LJ 3rd, Kan SH, Frye MA, Wahner HW, O'Fallon WM, Riggs BL (1989) Epidemiology of vertebral fractures in women. Am J Epidemiol 129(5):1000-1011

2. Majd ME, Farley S, Holt RT (2005) Preliminary outcomes and efficacy of the first 360 consecutive kyphoplasties for the treatment of painful osteoporotic vertebral compression fractures. Spine J $5: 244-255$

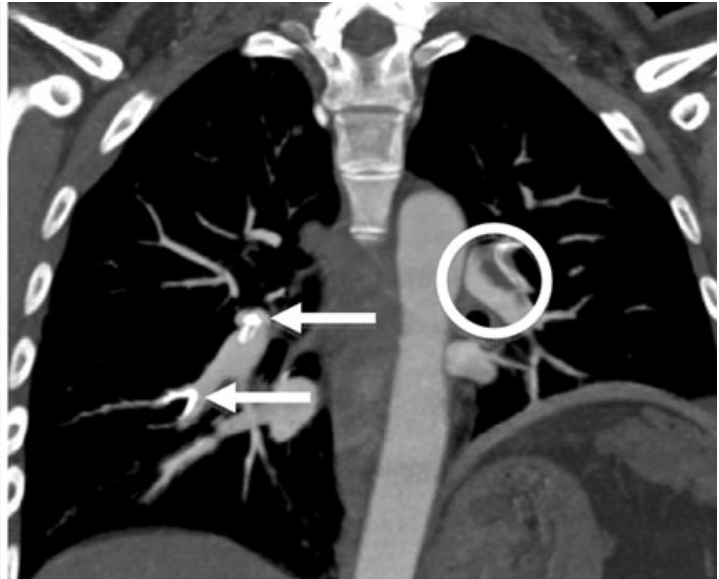

with associated thrombus at the bifurcation of the left upper and left lower lobar arteries (circles), all suggestive of pulmonary cement emboli from prior kyphoplasty

3. Lieberman IH, Dudeney S, Reinhardt MK et al (2001) Initial outcome and efficacy of "kyphoplasty" in the treatment of painful osteoporotic vertebral compression fractures. Spine 26:1631-1638

4. Choe DH, Marom EM, Ahrar K, Truong MT, Madewell JE (2004) Pulmonary embolism of polymethyl methacrylate during percutaneous vertebroplasty and kyphoplasty. Am J Roentgenol 183(4): 1097-1102

5. Krueger A, Bliemel C, Zettl R, Ruchholtz S (2009) Management of pulmonary cement embolism after percutaneous vertebroplasty and kyphoplasty: a systematic review of the literature. Eur Spine $\mathbf{J}$ 18:1257-1265 